Thorax, 1980, 35, 156-157

\title{
Pleuropulmonary complications of thoracic vertebral osteomyelitis
}

\author{
ROBERT BLOOM, HENRY YEAGER JR, AND VINCENT F GARAGUSI
}

From the Pulmonary Disease and Infectious Disease Divisions, Department of Medicine, Georgetown University Medical Center, Washington DC, USA

In the preantibiotic era, pyogenic thoracic vertebral osteomyelitis was often complicated by mediastinal abscess, empyema, or necrotising pneumonia which arose by contiguous invasion late in the disease and progressed to bacteraemia and death. ${ }^{1}$ Since the advent of antibiotics, however, the disease more often presents insidiously and may simulate mediastinal tumour, or gastrointestinal, genitourinary, or neurological disease. ${ }^{3}$ We have recently treated two patients with pleuropulmonary disease caused by pyogenic osteomyelitis involving thoracic vertebrae. In both, neoplasms were suspected initially and the true problem uncovered only after extensive investigation.

\section{Case reports}

CASE 1

A 49-year-old man presented with a six-week history of chills, fever, right pleuritic and right flank pain. He was thought to have bacterial pneumonia but failed to respond to oral antibiotics. Continued symptoms led to investigation for neoplasia, including gastrointestinal and gallbladder radiography, and a gallium scan. He was referred to Georgetown University Hospital. On admission, he was afebrile with tenderness over the lower thoracic vertebrae, dullness over the lower right lung field, and tubular breath sounds at both lung bases. His haematocrit was $36 \%$ with normal white cell count; Westergren sedimentation rate was $73 \mathrm{~mm} / \mathrm{hr}$. Chest radiograph revealed right basal atelectasis and pleural effusion, and blunting of the left costophrenic angle. Right thoracentesis yielded a straw coloured fluid with laboratory findings typical of a sterile exudate; pleural fluid sugar was $124 \mathrm{mg} / \mathrm{dl}$, and $\mathrm{pH}$ was 7.57. Pleural biopsy showed fibrinous pleuritis; pleural fluid cytology was negative.

On the third hospital day, a bone scan revealed increased radionuclide uptake at T10. Tomograms showed lytic lesions in T10 and T11 with narrowing of the disc space.

On the ninth hospital day, a left thoracotomy was performed and a paraspinous abscess located at T10 was evacuated. Cultures grew Staphylococcus aureus, and microscopic examination revealed inflammatory cells and reactive plasmacytosis. The patient was treated with nafcillin, 12 grams a day, for six weeks, and had an uneventful convalescence.

Address for reprint requests: Dr $\mathbf{H}$ Yeager Jr, Pulmonary Disease Division, Georgetown University Hospital, 3800 Reservoir Road, NW, Washington DC 20007, USA
CASE 2

A 63-year old man was admitted to hospital with two-month history of bilateral constricting lower thoracic pain. Chest radiograph and bone scan done one month before admission were normal. Five days before admission, the chest pain worsened and was accompanied by fever and anorexia.

He looked chronically ill with a temperature of $99 \cdot 8^{\circ}$. There was tenderness over the left lower rib? cage, and breath sounds were decreased over the lef lower lobe.

His haematocrit was $32 \%$, WBC 12800 per $\mathrm{mm}^{3} \vec{\bullet}$ Chest radiograph showed an apparent left paramedio astinal mass with hazy infiltration in the left lower lung field, and blunting of the left costophrenic angles (fig).

Fever continued during the next two weeks despite treatment with multiple antibiotics. Conventionaf thoracic spine films were normal, but tomograms re $\mathbb{D}$ vealed erosion of the vertebral plate at $\mathrm{T} 7$ and $\mathrm{T} 8$ with narrowing of the intervertebral space.

On the eighteenth hospital day, a left thoracotomy was performed and necrotic bone evacuated from the T7-T8 spinal area. Pathological examination showed. osteomyelitis and culture grew Staphylococcus aureus $\overrightarrow{5}$ Approximately $500 \mathrm{ml}$ of gelatinous fluid was drained from the left pleural space. Culture of the fluid wask negative, but Gram stain showed occasional Gramg positive cocci. Inflammatory changes were present in. the superior segment of the lower lobe adjacent to the involved vertebrae; lung biopsy revealed micro-3 abscesses from which Staphylococcus aureus was cultured.

After surgery, the patient was treated with nafcillin 12 grams per day intravenously for six weeks. He convalesced uneventfully except for mild persistenf bibasilar atelectasis by chest radiograph and the de velopment of a spinal curvature.

\section{Discussion}

The reasons for the rarity of overt pleuropulmonary complications in thoracic pyogenic osteomyelitis noves are unclear. Paraspinal abscesses may commonly migrate in a rostrocaudal direction in alignment with the potential space formed by the tissue planes of the posterior mediastinum and retroperitoneum. With this route available for decompression, we can hypothesis\& that anterior extension into the tighter confines of the pleural space is unlikely. In osteomyelitis of the ribs 


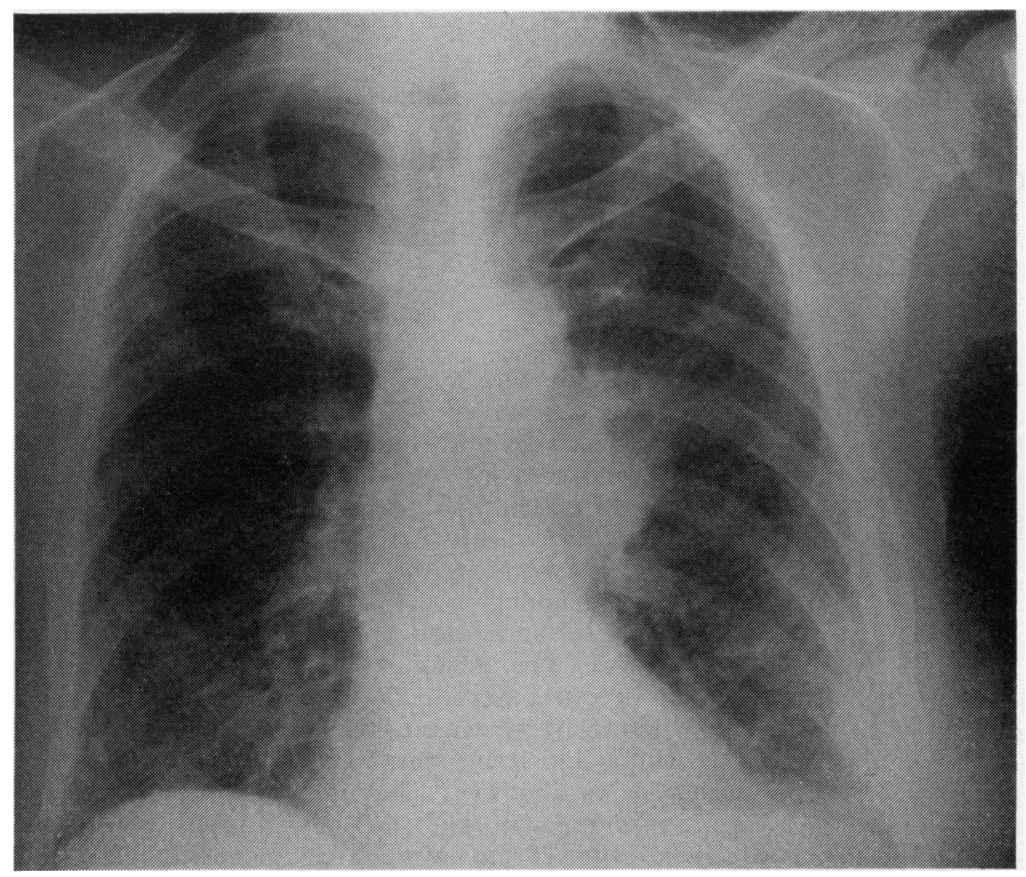

Chest roentgenogram of case 2 showing left paramediastinal mass, hazy infiltrate in the left lower lung field, and blunting of the left costophrenic angle.

by contrast, there is no space to decompress abscesses occurring adjacent to the bone, and, as a result, direct invasion of the pleura and lungs is common. ${ }^{4}$ Granulomatous infections of the thoracic vertebrae may more commonly present with bland pleural effusions, ${ }^{5}$ perhaps because these more indolent infections may progress further undiscovered than pyogenic infections. An additional possibility is that pleural effusions may accompany pyogenic vertebral osteomyelitis more commonly than is generally recognised. If no infection were present in the pleural space and lateral decubitus films were not examined, the effusion might be missed entirely or misinterpreted as nonspecific pleural thickening.

Pyogenic thoracic vertebral osteomyelitis should be considered in the differential diagnosis of unexplained paraspinal masses, pulmonary infiltrates, or pleural effusions, particularly if these abnormalities are accompanied by back pain and localised vertebral tenderness. Careful examination of the spine, radionuclide studies, tomograms, and ultimately biopsy of the affected area with appropriate bacteriological studies are important in establishing the presence of this treatable disease.

\section{References}

1 Wilensky AO. Osteomyelitis of the vertebrae. Ann Surg 1929; 89:561-70 and 731-47.

2 Waldvogel FA, Medoff G, Swartz MN. Osteomyelitis: a review of clinical features, therapeutic considerations and unusual aspects. $N$ Engl J Med 1970; 282:316-22.

3 Musher DM, Thorsteinsson SB, Minuth JN, Luchi RJ. Vertebral osteomyelitis. Arch Int Med 1976; 136:105-10.

4 Brock R. Osteomyelitis of the ribs. Guy's Hosp Rep 1957; 106:156-77.

5 Stevenson FH. The natural history of pleural effusion and orthopaedic tuberculosis. $J$ Bone Joint Surg 1955; 37B:80-91. 